\title{
Editorial
}

\section{Adolescente Embarazada, un tema aún pendiente en Chile.}

Para Chile y nuestra especialidad han sido un desafío, la Declaración de la Conferencia Mundial de Población en 1994, acerca de la Salud Sexual y Reproductiva,(SSR); que refrendó OMS en 2013 en Salud Sexual y Reproductiva Adolescente,(SSRA) y el texto de UNESCO de Guías Técnicas en Educación Sexual Integral Escolar (1,2,3).

La atención preferencial a las adolescentes embarazadas con programas dirigidos a la detección precoz de patologías y atención integral, se inician en la segunda década de los 60 , en Syracuse, con el auspicio de la Universidad de Nueva York y en el Estado de California apoyado por la Universidad de California. Se cambia el concepto de "primigesta precoz", considerado erróneamente como una entidad mórbida en sí misma y por lo tanto sin el concepto actual de Adolescente Embarazada. Situación en la cual se consideran variados factores de riesgo negativo que se pueden detectar, intervenir y prevenir en sus consecuencias (4).

En 1980 se inicia la aplicación de este enfoque, con intervención dirigida a la atención Integral de adolescentes embarazadas, en el Departamento de Obstetricia y Ginecología Norte de la Facultad de Medicina de la Universidad de Chile, en la antigua Maternidad del Hospital Universitario José Joaquín Aguirre. La experiencia se instaló progresivamente en la atención coordinada con los consultorios de atención primaria del Servicio de Salud Metropolitano Norte y fue apoyada por varios proyectos de investigación de concursos y de donaciones de Agencias Internacionales como IPPF y Fundación Ford (5).

El Ministerio de Salud, a partir de 1990, implementa progresivamente programas para adolescentes en el sistema de Atención Primaria con la participación de jóvenes de la comunidad.

Los impactos en salud se apreciaron en los programas con intervención, sin embargo, la tasa de fecundidad adolescente recién inicia su descenso 22 años después, en 2012-2013, en el grupo de 15-19 años. El grupo de 10 a 14 años muestra un leve descenso recién en 2016.

El impacto de este enfoque fue medido en un estudio de seguimiento de adolescentes embarazadas, con y sin morbilidad y determinó que los mayores factores de riesgo asociados fueron: La ausencia del progenitor, una estatura de la adolescente de 1,50m o menos, embarazo ocurrido por seducción o violación, tener sólo dos años o menos de educación media, edad de la Menarquia a los 11 años o menos, estado de nutricional enflaquecida y una actitud negativa o indiferente, de la adolescente. Este estudio de seguimiento de 6 años fundamentó la evidencia para la atención a adolescentes embarazadas, según niveles de complejidad en la Atención en Salud (6). Sin embargo, esta experiencia no se expandió al resto del País.

Los autores del trabajo "Embarazo Adolescente en Chile: Una mirada a la desigualdad socio demográfica comunal", que se incluye en el presente número, encuntran diferencias comunales en la fecundidad adolescente en Chile. Estas diferencias podrían ser explicadas por el mayor riesgo de embarazo en las adolescentes de las comunas con mayor desigualdad socioeconómica donde no se aplican programas diferenciados de atención a adolescentes embarazadas que necesitan recursos de mayor complejidad. En estos grupos de mayor riesgo es indispensable aplicar la atención por Niveles de Complejidad, especialmente en las comunas más pobres. A su vez importante, la coordinación entre los niveles Primario y Secundario de la Atención de las adolescentes embarazadas.

A la Atención en Salud hay que agregar el aporte de la Educación, que es fundamental en el control de la fecundidad, tanto deseada, no deseada como inesperada. Se conoce el aporte evidente de la Educación Sexual Integral Escolar (ESIE), en todos los estudios de prevención del embarazo adolescente $(7,8)$. La ESIE, no se aplica en Chile hasta el día de 
hoy, como ocurre en los países desarrollados y en todo el resto de los países de la Región. Según UNESCO, es una herramienta fundamental en la prevención del Embarazo Escolar (9).

La existencia y calidad en la aplicación de esta intervención: ESIE, es significativamente diferente según el nivel socioeconómico de las comunas.

En la actual discusión para legislar sobre la Ley de Educación Sexual Escolar en el Congreso de la República, se plantean varias alternativas para la cobertura del programa, según niveles escolares. Sin embargo, es preocupante que la estrategia actual del MINEDUC, se base en la entrega de herramientas metodológicas para que cada establecimiento escolar diseñe sus programas de Educación Sexual, aparentemente para proteger la diversidad cultural. Esto resulta preocupante considerando que no más de un $10 \%$ de los profesores en Chile, tiene capacitación en ESIE y que estos se concentran en la educación privada. Un programa de Educación Sexual es complejo en el diseño, aplicación y evaluación de calidad e impacto. Los primeros resultados de esta experiencia, presentados en la Comisión de Educación de la Cámara de Diputados, mostraron que la proporción de programas diseñados ha sido baja pero, significativamente más alta en el sector privado de la educación. No se conocen experiencias semejantes de esta estrategia en otros países, donde son únicos o diversos programas ya diseñados, con la experticia de profesionales de la educación que se ofrecen a los establecimientos escolares.

Chile por ser el país más largo del mundo, necesita herramientas de capacitación a distancia para avanzar en esta materia y con modelos pedagógicos probados que estén a libre disposición y sean de fácil aplicación en la educación publica y privada.

Los sectores de salud y educación podrían contribuir a la disminución del impacto de la inequidad entre las comunas que se reflejan en este estudio de Fecundidad Adolescente, a través de la Atención de Adolescentes Embarazadas por niveles de complejidad del Riesgo y a través de la Educación Sexual Integral Escolar, gratuita, ya probada (10).

\section{Prof. Dr. Ramiro Molina Cartes Profesor Emérito Universidad De Chile.}

\section{Bibliografía}

(1) United Nations. Report of the International Conference on Population and Development, Document A/Conf. 171/13, New York, , 1994, paragraph 7.2.

(2) Chandra-Mouli, Bloem P, Ferguson J. 2013 The World Health Organization's work on adolescent sexual and reproductive health. doi: 10.1007/s00103-012-1606-0.

(3) UNESCO, 2018. International technical guidance on sexuality education: Introduction page 12, Section 4: The evidence base for comprehensive sexuality education, pages 28-31.

(4) Osofsky, H.J., Osofsky, J.D., Kendall, N. et al. 1973. Adolescents as mothers: An interdisciplinary approach to a complex problem. J. Youth Adolesc. 2:233. Doi: https://doi.org/10.1007/BF02213859

(5) R. Molina; M.I. Romero y cols. 1985 Adolescente Embarazada. Resultado de un modelo de atención médica. Rev. Med. de Chile. 113: 154- 161.

(6) Molina Ramiro, González Electra. 2012. Teenage Pregnancy, In Charles Sultan (Ed.) Pediatric and Adolescent Gynecology. Evidence-Based clinical Practice, 2nd. Revised and extended edition. Endocr. Dev. Basel, Karger, vol 22, pp 302-331.

(7) Dan Apter, Ramiro molina C., Sexuality Education: Finnish and Chilean Experiences. In Charles Sultan (Ed.). 2012. EvidenceBased clinical Practice, 2nd. Revised and extended edition. Endocr. Dev. Basel, Karger, vol 22, pp 332-356.

(8) Kirby, D, Rolleri, L \& Wilson, MM. (2007). Tool to Assess the Characteristics of Effective Sex and STD/HIV Education Programs. Washington, DC: Healthy Teen Network.

(9) UNESCO, 2016. Review of the Evidence on Sexuality Education. Report to inform the update of the UNESCO International Technical Guidance on Sexuality Education; prepared by Paul Montgomery and Wendy Knerr, University of Oxford Centre for Evidence-Based Intervention. Paris, UNESCO.

(10) Curso de Educación Sexual On Line de Auto Aprendizaje (CESOLAA). Disponible en: www.educacionsexual.uchile.cl 